\title{
Determination of the static scaling exponent of self-affine interfaces by nonspecular $x$-ray scattering
}

\author{
T. Salditt, T. H. Metzger, Ch. Brandt, U. Klemradt, and J. Peisl \\ Sektion Physik der Ludwig-Maximilians-Universität München, Geschwister-Scholl-Platz 1, 80539 München, Germany
}

(Received 6 September 1994; revised manuscript received 7 November 1994)

\begin{abstract}
We discuss the conditions that have to be met in a nonspecular x-ray experiment to extract the static scaling behavior of a self-affine surface independently of a special height-height correlation function chosen to model the data. It is shown that this task requires a sufficiently large parallel momentum transfer of the scattering vector. In this respect, various scattering geometries are compared, and an analytical approximation to the structure factor on the relevant range of the scattering vector is proposed. The validity of the approximation is checked numerically.
\end{abstract}

\section{INTRODUCTION}

In a seminal paper, Sinha et al. have studied the x-ray scattering cross section of randomly rough surfaces, characterized by an arbitrary height-height correlation function $c(r)$, where $r$ is the distance between two points on the surface. ${ }^{1}$ They have derived the scattering cross section at glancing incidence and exit angles within the distorted-wave Born approximation (DWBA). The resulting structure factor $S(\mathrm{q})$ is an integral transform of the function $c(r)$ for which in general no analytical expression exists. Due to the statistical averaging inherent to the scattering process, $\mathrm{x}$-ray scattering is a well-suited tool to deduce quantitative information on surface morphology, and the DWBA has been applied many times to analyze experimental data and to determine certain parameters of the height-height correlation function. ${ }^{2-4}$

Special interest has been placed in the study of selfaffine surfaces, which have been predicted theoretically to result from various growth processes far from thermodynamic equilibrium. ${ }^{5}$ In this case, the asymptotic behavior of $c(r)$ is governed by a fundamental scaling law, i.e., for $r$ much smaller than an upper cutoff length $\xi$ the function $c(r)$ decays by a power law with an exponent $H$ referred to as the static scaling exponent or wandering exponent. This exponent is determined by universal properties like dimension, symmetry, and conservation laws. Thus, in order to study the scaling behavior, a scattering experiment has to be performed in a manner that the region of reciprocal space probed is sensitive to the range $r<<\xi$ in real space. This point will be elucidated in the present work, as well as the related question on the choice of the correlation function $c(r)$ to be used in the structure factor when fitting experimental data. ${ }^{6} \mathrm{We}$ will further discuss an analytical approximation to $S(\mathrm{q})$ of self-affine surfaces that is valid in the limit of weak roughness, $\left|q_{z}^{\prime} \sigma\right|^{2} \ll 1$, where $\sigma$ is the rms roughness of the surface and $q_{z}^{\prime}$ is the normal component of the scattering vector.

The paper is organized as follows: In Sec. I, a brief outline of the theory of kinetic roughening is given, following closely the approach of Krug and Spohn. ${ }^{5}$ The ma- terial presented in this section is not new. However, with respect to the great significance for the following sections and for the sake of notational clarity, a coherent, short summary of the underlying ideas might be helpful. Several possible forms of the height-height correlation function (also termed scaling function in the theoretical context) are presented. Section II is concerned with the structure factor of a rough surface as derived within the DWBA. It is shown that $S(\mathrm{q})$ decays by a power of $\gamma$ in the range of parallel momentum transfer $q_{\|} \gg 2 \pi / \xi$. From this exponent $\gamma$ the static scaling exponent $H$ can be determined independently of the detailed form of $c(r)$, as long as the asymptotic behavior is fixed. In the limit of weak roughness the structure factor reduces to the Fourier transform of $c(r)$ and $\gamma=2+2 H$. The validity of this approximation is checked numerically for various parameters. In Sec. III, different scattering geometries conventionally used for nonspecular $\mathrm{x}$-ray measurements as well as out of the plane of reflection-geometry are discussed. Section IV is concerned with the determination of $H$ in a multilayer system. Finally, a summary and some conclusions are presented in Sec. V.

\section{SELF-AFFINE ROUGHNESS}

Let us consider a growing surface on a scale that is much smaller than the macroscopic sample size and yet large compared to the microscopic length scale of the system, e.g., the interatomic distance $a$. The growth process is defined by some microscopic mechanism that determines how the new material is deposited on the surface and how it is incorporated into the aggregate. This will be accompanied by random noise, e.g., in the flux or aggregation rates, and will thus lead to a roughening of the surface. After subtraction of the average height, we can parametrize the rough surface by a single-valued, continuous height function $h(\mathbf{r}, t)$. Starting from a perfectly flat substrate at time $t=0$, fluctuations will build up of growing lateral and normal extensions. Let us define $\xi_{\|}(t)$ to be the largest present lateral correlation length of such a fluctuation at a given stage of growth and let $\xi_{\perp}(t)$ be the corresponding normal correlation length, i.e., the typical length scales of the largest bumps or cusps that 
have developed.

Let us further assume that the stochastic process defined by the growth introduces no additional length scales, e.g., no diffusion length. For lateral and normal length scales smaller than $\xi_{\|}$and $\xi_{1}$, respectively, the statistical properties of the growing surface are then not expected to change after scaling the system, but should instead depend only on dimension, symmetry, and conservation laws, in analogy to the theory of critical phenomena. Thus, naively one is tempted to assume that the surface "looks alike" on all length scales, i.e., a self-similar or fractal structure. However, the substrate dimensions (parallel to the surface) and the dimension normal to the average surface are not equivalent and thus cannot be scaled by the same factor. Instead of self-similarity, one should assume self-affinity taking into account the difference between the parallel and the normal dimensions. If the lateral position vector $\mathbf{r}$ is scaled by a factor $b, h(\mathbf{r}, t)$ by $b^{-H}$, and $t$ by $b^{z}$, the rescaled surface $\widetilde{h}$

$$
\tilde{h}(\mathbf{r}, t)=b^{-H} h\left(b \mathbf{r}, b^{z} t\right),
$$

should itself be a realization of the same stochastic process, i.e., $\widetilde{h}$ and $h$ should possess the same statistical expectation values. This is the basic scaling hypothesis. The exponents $H$ and $z$ characterize the surface fluctuations and are determined by the corresponding growth process. In Eq. (2.1) we impose the condition $z>0$ and $0<H<1$, which will be justified below.

To elucidate the implications of this scaling behavior, let us consider the squared height difference correlation function of an isotropical surface

$$
g\left(\left|\mathbf{r}-\mathbf{r}^{\prime}\right|, t\right)=\left\langle\left[h(\mathbf{r}, t)-h\left(\mathbf{r}^{\prime}, t\right)\right]^{2}\right\rangle .
$$

This quantity is the average squared height difference of two points $\mathbf{r}$ and $\mathbf{r}^{\prime}$ on the surface separated laterally by $r:=\left|\mathbf{r}-\mathbf{r}^{\prime}\right|$. Inserting Eq. (2.1) in Eq. (2.2) we obtain

$$
g(r, t)=b^{-2 H} g\left(b r, b^{z} t\right),
$$

and by setting $b=1 / r$

$$
g(r, t)=r^{2 H} g\left(1, t / r^{z}\right)=: r^{2 H} g\left(t / r^{z}\right) .
$$

In the limit $t \rightarrow \infty, g(r, t)$ is supposed to become stationary, and the height difference correlation will only be a function of the length scale $r$ on which it is measured,

$$
g(r, t) \propto r^{2 H} .
$$

Rough surfaces are characterized by $0 \leq H \leq 1$. The limiting case $H=1$ implies that the surface has no longer a macroscopic orientation parallel to the substrate, as $\sqrt{g(r)} / r$ does not vanish for $r \rightarrow \infty$. In the other limit $H=0$ the power law of Eq. (2.5) is replaced by a logarithmic behavior

$$
g(r, t) \propto \ln (r) .
$$

If the roughness fluctuations have not yet become stationary on a given length scale $r^{\prime}$, i.e., $t$ is much smaller than the time that is necessary to build up fluctuations on the length $r^{\prime}$, the height difference correlation should increase with time according to $g(r, t) \simeq t^{2 \beta}$ independently of $r \leq r^{\prime}$. We thus impose the following asymptotic behavior of the scaling function $g(x)$, Eq. (2.4):

$$
g(x) \simeq\left\{\begin{array}{l}
x^{2 \beta} \text { for } x<1, \\
\text { const for } x \gg 1
\end{array}\right.
$$

with $\beta=H / z$. The crossover $g(x \simeq 1)$ between the two asymptotic regimes is given by $t \simeq r^{z}$, see Eq. (2.4). Correspondingly, the lateral correlation length at a given time is determined by this condition to be $r \simeq t^{1 / z}$. At $g(x \simeq 1)$ the scaling function is generally not known. A typical function $g(r)$ fulfilling the asymptotic constraints of Eq. (2.7) is plotted in Fig. 1.

Summarizing Eq. (2.7), the lateral correlation length grows as $\xi_{\|} \propto t^{1 / z}$, whereas the normal correlation length increases according to $\xi_{\perp} \propto \xi_{\|}^{H} \propto t^{H / z}$, and the height difference correlation function obeys $g(r, t) \propto r^{2 H}$ for $r<<\xi_{\|}$until it becomes constant at $r \gg \xi_{\|}$.

How does the existence of a finite sample size $L$ influence this scaling behavior? The answer is simply that growth will become stationary as soon as the maximum lateral correlation length $\xi \simeq L$ has developed. In the following, by the correlation length $\xi$ we will always understand the lateral correlation length $\xi_{\|}$. However, before the sample size becomes relevant, for a real system there may be a still smaller upper cutoff length that can be introduced by the growth itself or be the result of a macroscopic surface treatment. Finally, in an experiment, the measuring technique itself can in some cases restrict the fluctuations probed to be smaller than a value $\xi$, i.e., by a finite coherence length in a scattering experiment.

The scaling hypothesis and self-affine surface roughness have been verified in a number of computer simulations. ${ }^{7}$ They are the fundamental concepts in the theory of kinetic roughening, by which the scaling exponents of a rough surface can be predicted from the microscopic features of a growth model. This task has so far only been achieved for a restricted class of models, where the normal velocity of the growing surface at a particular point is governed by the local derivatives of the height function. For this case, Kardar, Parisi, and Zhang (KPZ)

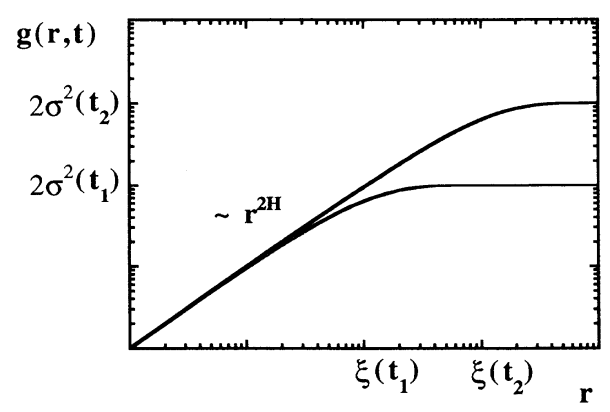

FIG. 1. The asymptotic regimes of the height difference function $g(r, t)$ are shown as a function of $r$ for two times $t_{1} \leq t_{2}$ : At $r \ll \xi(t), g(r, t)$ increases by a power of $2 H$ before saturating to a value of $2 \sigma^{2}(t)$ for $r \gg \xi(t)$. 
have shown in a classical paper that the evolution of $h(\mathrm{x}, t)$ is governed by the following Langevin equation: ${ }^{8}$

$$
\frac{\partial h(\mathbf{r}, t)}{\partial t}=v \nabla^{2} h(\mathbf{r}, t)+\lambda[\nabla h(\mathbf{r}, t)]^{2}+\eta,
$$

where $v$ is an efficient surface stiffness, $\lambda$ is the nonlinear coefficient, and $\eta$ a random white noise. For this equation, numerical studies have revealed an exponent $H \simeq 0.39$ in $2+1$ dimensions. $^{9}$

Now consider a self-affine surface, that has evolved in a certain growth process and is assumed to stay in the corresponding metastable state. It can be characterized by a roughness exponent $H$ and an upper cutoff $\xi \gg a$. In this case, a simple form of the height difference correlation function would be

$$
g(r)=2 \sigma^{2}\left\{1-\exp \left[-(r / \xi)^{2 H}\right]\right\} .
$$

Clearly, for $r \ll \xi$ this function fulfills the scaling assumption, and for $r \geq \xi$ the height difference correlation saturates to a value of $2 \sigma^{2}$, where $\sigma$ is the rms roughness. However, in between the asymptotic limits, the scaling hypothesis does not justify the specific choice of Eq. (2.9) or any other functional form. In contrast to the universal roughness exponents, the form of the function $g(r)$ at $r \simeq \xi$ is not predicted by theory. In principle, the scaling function $g(r)$ is expected to be a universal quantity, but its exact form is not known. Thus, it is primarily its asymptotic behavior that should be tested experimentally.

By the identity

$$
\begin{aligned}
\left\langle\left[h(\mathbf{r})-h\left(\mathbf{r}^{\prime}\right)\right]^{2}\right\rangle= & \left\langle h(\mathbf{r})^{2}\right\rangle+\left\langle h\left(\mathbf{r}^{\prime}\right)^{2}\right\rangle \\
& -2\left\langle h(\mathbf{r}) h\left(\mathbf{r}^{\prime}\right)\right\rangle
\end{aligned}
$$

and by translation invariance we obtain the height-height correlation function corresponding to Eq. (2.9):

$$
c(r):=\left\langle h(\mathbf{r}) h\left(\mathbf{r}^{\prime}\right)\right\rangle=\sigma^{2} \exp \left[-(r / \xi)^{2 H}\right] .
$$

Accordingly, we find for the two asymptotic cases

$$
c(r) \simeq\left\{\begin{array}{l}
\sigma^{2}\left[1-(r / \xi)^{2 H]} \text { for } r<<\xi,\right. \\
0 \text { for } r>\xi \xi .
\end{array}\right.
$$

Due to its simple mathematical form, Eq. (2.11) has been used to calculate the $x$-ray structure factor of a rough surface. ${ }^{1}$ Other functions that have been studied in the context of nonspecular $\mathrm{x}$-ray scattering are ${ }^{6}$

$$
\begin{aligned}
& c(r)=\sigma^{2}\left[1-\tanh ^{2 H}(r / \xi)\right], \\
& c(r)=\sigma^{2}\left\{1-e^{-(r / \xi) H}[2 \cosh (r / \xi)-2]^{H}\right\}, \\
& c(r)=\sigma^{2}\left(1-\left[1-e^{-(r / \xi)^{2}}\right]^{H}\right) .
\end{aligned}
$$

The functions, Eqs. (2.12)-(2.15) all fulfill the scaling requirements of Eq. (2.4). However, they can only describe surfaces with roughness exponents $0<H \leq 1$, but do not contain the case of logarithmic roughness " $H=0$." For $H=0$ they become constant which is not physically meaningful. The height difference function of Eq. (2.9) is plotted in Fig. 2 for small values of $H$ in dimensionless units $R:=r / \xi$ and $G(R):=g(r) / 2 \sigma^{2}$. Throughout the

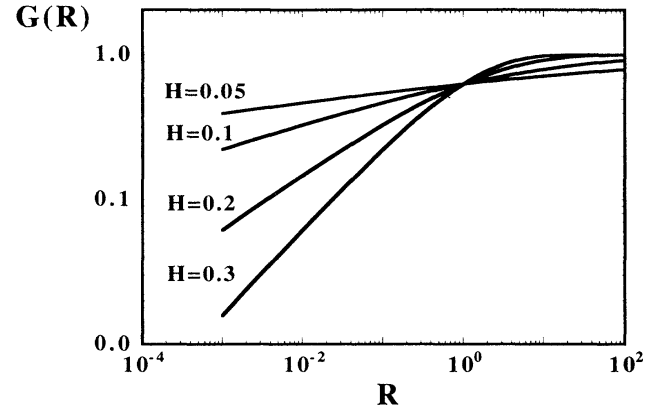

FIG. 2. The height difference function of Eq. (2.9) for values of $H=0.3,0.1,0.05$, in dimensionless units $R:=r / \xi$ and $G(R):=g(R) /(2 \sigma)^{2}$. As can be seen, the power-law regime is restricted to smaller and smaller $R$ in the limit $H \rightarrow 0$ and the transition regime $r \simeq \xi$ becomes ill defined. Equation (2.12) is thus not an appropriate correlation function for small values for $H$ in the sense of the scaling hypothesis.

manuscript the capital letters, $R, G(R), C(R)$, and $Q$ are used for dimensionless quantities to be defined in the text. As can be seen, the asymptotic region $r \leq \xi$ where $g(r) \propto r^{2 H}$ according to self-affine roughness is vanishing in the limit $H \rightarrow 0$. This is a particular feature of Eq. (2.9), which shows that this function is not appropriate in the limit $H \rightarrow 0$.

To overcome this drawback, the so-called $K$ correlation function has recently been proposed to describe self-affine surfaces. ${ }^{10}$

$$
c(r)=\frac{2 H \sigma^{2}}{\Gamma(1+H)}\left(\frac{r \sqrt{2 H}}{2 \xi}\right) K_{H}\left(\frac{r \sqrt{2 H}}{\xi}\right),
$$

where $K_{H}$ is the Bessel function of second kind and of order $H$, and $\Gamma$ denotes the $\gamma$ function. This function has a logarithmic behavior in the limit $H \rightarrow 0,{ }^{10}$ i.e., is of the form

$$
c(r)=A-B \ln r .
$$

The height difference function corresponding to Eq. (2.16) is shown together with those of Eq. (2.13)-(2.15) and with Eq. (2.9) for $H=0.5$ in Fig. 3. Indeed, on the $\log -\log$ plot the asymptotic scaling behavior is found to be the same for all curves, while in the linear inset different forms for $r \simeq \xi$ are clearly visible.

A further advantage is the fact that Eq. (2.16) has an analytical Fourier transformation, i.e., an analytical spectral power density or roughness spectrum. For $H \neq 0$ it is given by

$$
\left\langle|z(\mathbf{k})|^{2}\right\rangle=\frac{L_{x} L_{y}}{(2 \pi)^{5}} \frac{\sigma^{2} \xi^{2}}{\left(1+u k^{2} \xi^{2}\right)^{1+H}},
$$

where $L_{x} L_{y}$ is the area over which Eq. (2.16) has been integrated, i.e., the illuminated area in a scattering experiment. The constant $u$ has to be determined from the implicit equation

$$
u=\frac{1}{2 H}\left\{1-\left[1+u(\pi / a)^{2} \xi^{2}\right]^{-H}\right\} .
$$




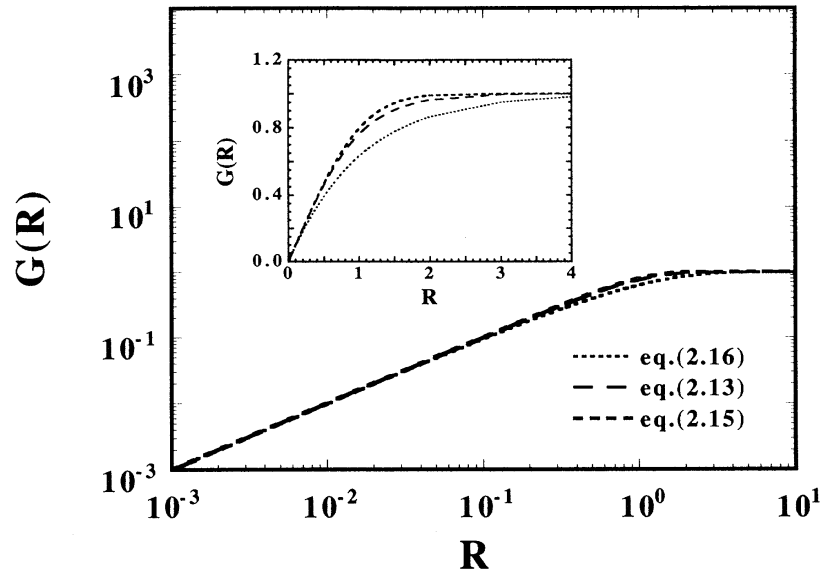

FIG. 3. The height difference functions corresponding to Eqs. (2.13), (2.15), and (2.16) with the same asymptotic behavior $(H=0.5)$ are shown on a log-log scale and on a linear scale (inset), respectively. For $H=0.5$, Eqs. (2.12) and (2.16) are equal. The difference between Eqs. (2.14), (2.12), and (2.16) is negligible.

If the ratio between upper and lower cutoff $\xi / a$ is large, $u \rightarrow 1 / 2 H$. In the case $H=0$ the Fourier transform of Eq. (2.16) reads

$$
\left\langle|z(\mathbf{k})|^{2}\right\rangle=\frac{L_{x} L_{y}}{(2 \pi)^{5}} \frac{\sigma^{2} \xi^{2}}{1+u k^{2} \xi^{2}},
$$

where $u$ is determined form

$$
u=0.5 \ln \left[1+u(\pi / a)^{2} \xi^{2}\right]
$$

\section{STRUCTURE FACTOR OF A ROUGH SURFACE}

Nonspecular (diffuse) $x$-ray scattering is a technique, where the intensity scattered from a rough interface is measured at grazing incident and exit angles close to the critical angle for total external reflection with the detector moved out of the specular position. Let the $z$ axis be parallel to the average normal vector of the height function $h(x, y)$, and let the plane of reflection be the $x z$ plane. If $\mathbf{k}_{\mathbf{i}}$ and $\mathbf{k}_{\mathbf{f}}$ denote the initial and the final vacuum wave vectors, both of magnitude $k=2 \pi / \lambda$, the scattering vector is defined by $\mathbf{q}=\mathbf{k}_{\mathbf{f}}-\mathbf{k}_{\mathbf{i}}$, and the specular condition is given by $q_{x}=q_{y}=0, q_{z}>0$. In the medium, characterized by a complex index of refraction $n$, the $q_{z}$ is changed to $q_{z}^{\prime}$ according to Snell's law. Diffuse intensity is measured with a parallel momentum transfer component $q_{\|}$, that can be $q_{x}, q_{y}$, or a superposition of both. In contrast to specular reflectivity, from which the average electron density profile along the interface normal can be deduced, the diffuse intensity contains information on the lateral structure of rough interfaces. Sinha et al. have shown ${ }^{1}$ that within the distorted-wave Born approximation the differential cross section for $x$-rays scattered from a rough interface is given by

$\frac{d \sigma}{d \Omega}=\left(L_{x} L_{y}\right) \frac{\pi^{2}}{\lambda^{4}}\left(1-n^{2}\right)^{2}\left|T\left(\mathbf{k}_{\mathrm{i}}\right)\right|^{2}\left|T\left(\mathbf{k}_{\mathrm{f}}\right)\right|^{2} S\left(\mathbf{q}^{\prime}\right)$,

where $L_{x} L_{y}$ is the illuminated area on the sample, $T(\mathbf{k})$ is the Fresnel transmission function and $S\left(q^{\prime}\right)$ is the structure factor. The transmission functions

$$
T\left(\mathbf{k}_{\mathbf{i}, \mathrm{f}}\right)=\frac{2 \sin \alpha_{i, f}^{\prime}}{\sin \alpha_{i, f}+n \sin \alpha_{i, f}^{\prime}},
$$

with $\alpha_{i, f}$ being the grazing angle of incidence and exit, respectively, and $\alpha_{i, f}^{\prime}$ the corresponding angles of refraction, describe how the incoming wave penetrates the vacuum-medium interface before scattering and how the scattered wave leaves the medium again after being scattered from lateral roughness components. The maximum of $T\left(\mathbf{k}_{\mathrm{i}, \mathrm{f}}\right)$ for $\alpha_{i, f}=\alpha_{c}$ gives rise to the so-called Yoneda peaks in the diffuse scattering, ${ }^{11} \alpha_{c}$ being the critical angle of total external reflection. The Fresnel transmission functions have a drastic effect on the measured intensity, but are "optical" in nature and do not stem from interfacial roughness. However, it has been argued that the transmission functions have to be modified by the rms roughness $\sigma$ according to $T(\mathbf{k}) \exp \left[q_{z} q_{z}^{\prime} \sigma^{2}\right] .{ }^{12}$ Information on the height-height correlation function $c(r)$ is contained in the structure factor

$$
S\left(q_{z}^{\prime}, q_{x}, q_{y}\right)=\frac{\exp \left[-\operatorname{Re}\left(q_{z}^{\prime}\right)^{2} \sigma^{2}\right]}{\left|q_{z}^{\prime}\right|^{2}} \int_{0}^{\infty} d r r\left\{\exp \left[\left|q_{z}^{\prime}\right|^{2} c(r)\right]-1\right\} J_{0}\left(q_{\|} r\right)
$$

where $J$ denotes the Bessel function of first kind. Often the nonspecular intensity is integrated in one direction, e.g., along $q_{y}$ by means of a wide open slit directed parallel to the $y$ axis. The structure factor as a function of only $q_{x}$ then takes the form

$$
S\left(q_{z}^{\prime}, q_{x}\right)=\frac{\exp \left[-\operatorname{Re}\left(q_{z}^{\prime}\right)^{2} \sigma^{2}\right]}{\left|q_{z}^{\prime}\right|^{2}} \int_{0}^{\infty} d r\left\{\exp \left[\left|q_{z}^{\prime}\right|^{2} c(r)\right]-1\right\} \cos \left(q_{x} r\right) .
$$

We will examine this result with the following questions in mind: (1) How can the static roughness exponent $H$ be deduced from $S\left(\mathbf{q}^{\prime}\right)$, independently of a special functional form of $c(r)$ ? (2) Under which conditions can the integral of Eq. (3.3) be approximated by a Fourier transform of $c(r)$, i.e., when is the so-called approximation of "weak roughness" valid? First, let us rewrite the structure factor of Eq. (3.3) in reduced variables, $Q:=q_{\|} \xi$ for the dimensionless parallel momentum transfer, and $R:=r / \xi$ for the dimensionless distance in real space,

$$
S\left(Q, q_{z}^{\prime}\right)=F\left(q_{z}^{\prime}\right) \xi^{2} \int_{0}^{\infty} d x \times\left\{\exp \left[-\left|q_{z}^{\prime}\right|^{2} \sigma^{2} C(x / Q)\right]-1\right\} J_{0}(Q),
$$


where $x:=Q R, C(R):=c(R) / \sigma^{2}$, and the factor in front of the integral in Eq. (3.3) has been abbreviated by $F\left(q_{z}^{\prime}\right)$. As can be seen, the structure factor is proportional to the square of the lateral cutoff $\xi$ and to the factor $F\left(q_{z}^{\prime}\right):=\exp \left[\operatorname{Re}\left(q_{z}^{\prime}\right)^{2} \sigma^{2}\right] /\left|q_{z}^{\prime}\right|^{2}$. Hence, $\left|q_{z}^{\prime}\right|$ has to be kept small in order to get a large intensity. Figure 4 shows $\left|q_{z}^{\prime}\right|$ as a function of $\alpha_{i}=\alpha_{f}$. It is clear that a measurement with the incidence and exit angles equal the critical angle gives the highest diffuse intensity.

In general, there is no analytical solution to the integral of Eq. (3.5), and the numerical treatment with $C(R)$ given by Eqs. (2.11)-(2.15) and in particular Eq. (2.16) is of very slow convergence, and moreover quite unstable for some integration algorithms. Calculated curves for four different exponents $H$ at constant $q_{z}^{\prime}$ with $C(R)$ according to Eq. (2.11) are presented in Fig. 5 on a $\log -\log$ scale. Quite similar to the Guinier and Porod region, respectively, of conventional small angle scattering, the structure factor is essentially constant for $Q \leq 1$ and decays with a power of $\gamma$ for $Q \gg 1$. Therefore, the value of $\xi$ can be experimentally determined from the change of slope in the log-log plot of the structure factor. The static scaling exponent $H$ can be deduced from $\gamma$, as $\gamma$ is a function of $H$ and of $\left|q_{z}^{\prime}\right| \sigma$,

$$
S(Q) \propto Q^{-\gamma}, Q \gg 1, \quad \gamma=f\left(H, q_{z} \sigma\right) .
$$

Only in the case of a logarithmic correlation function, i.e., Eq. (2.17), we know a general analytical expression for the exponent $\gamma$, i.e., the expression derived within the Born approximation [Eq. (2.38) of Ref. 1]

$$
\gamma=2+2 H-\left(\left|q_{z}\right| \sigma\right)^{2} .
$$

Numerical calculation shows that Eq. (3.7) still holds within the DWBA. Equation (3.6) can be checked by calculating the structure factor for different correlation functions $C(R)$. However, the results for $\gamma$, and hence for $H$, are found to be independent of the special form of

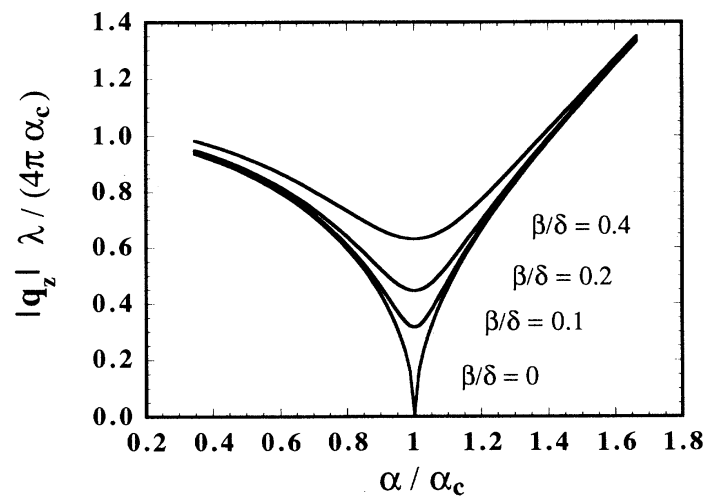

FIG. 4. The modulus of the complex, normal momentum transfer $\left|q_{z}^{\prime}\right|$ in a medium of index of refraction $n=1-\delta-i \beta$ in dimensionless units as a function of incidence and exit angle $\alpha:=\alpha_{i}=\alpha_{f}$. Curves for values of $\beta / \delta=0,0.1,0.2,0.4$, respectively, are shown from bottom to top.

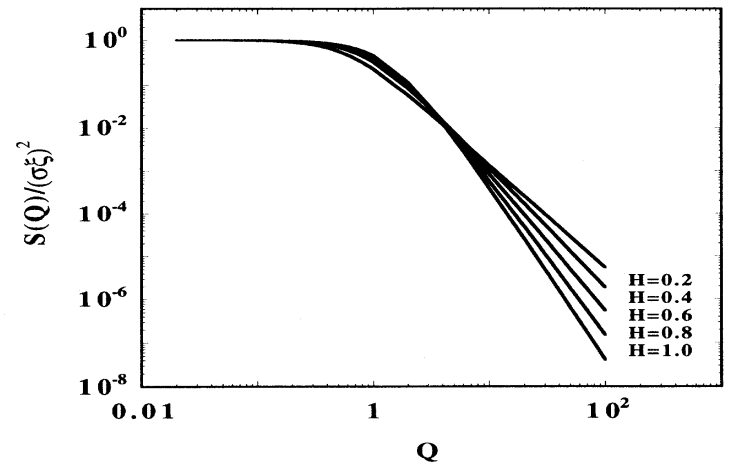

FIG. 5. Structure factor $S\left(\left|q_{z}^{\prime}\right|=\right.$ const, $\left.Q\right)$ calculated from Eq. (2.16) in the limit of weak roughness, $\left(\left|q_{z}^{\prime}\right| \sigma\right)^{2} \rightarrow 0$, for different values of $H$.

$C(R)$, as long as the scaling behavior is correct. The values of $\xi$ determined by this procedure are found to be approximately the same. As a consequence of this numerical result, one must make sure to determine $H$ from data fitted at high $Q$ and not at $Q \leq 1$ where the result would depend on the exact form of $C(R)$.

To answer the second question raised above, we have calculated $S(Q)$ for different values of $\left(\left|q_{z}^{\prime}\right| \sigma\right)^{2}$ using $C(R)$ as given by Eq. (2.16). We can compare the results to the analytical Fourier transformation, Eq. (2.18), which is an approximation to the exact structure factor of Eq. (3.3). It has been pointed out that this approximation should hold for $\left(\left|q_{z}^{\prime}\right| \sigma\right)^{2} \ll 1,{ }^{1}$ since in this case a Taylor expansion of the exponential is justified. However, not only $\left(\left|q_{z}^{\prime}\right| \sigma\right)^{2}$, but also $C(R)<1$ can reduce the exponential, and the approximation can thus be expected to hold even at $\left(\left|q_{z}^{\prime}\right| \sigma\right)^{2} \leq 1$. It was therefore of interest to check the approximation numerically also in this range. By a Taylor expansion to first order, the $\left(\left|q_{z}^{\prime}\right| \sigma\right)^{2}$ dependence of the integral in Eq. (3.3) vanishes and the exponent $\gamma$ will be independent of $\left(\left|q_{z}^{\prime}\right| \sigma\right)^{2}$ in this limit. The numerical calculation of the Fourier transform approximation yielded in all cases $\gamma=2+2 \mathrm{H}$, as expected from the analytical expression, Eq. (2.18). To obtain the exact structure factor, Eq. (3.5) was integrated numerically for $\left(\left|q_{z}^{\prime}\right| \sigma\right)^{2}=0.1,0.5,1$, in the range $1<Q \leq 100$, and on the range $10 \leq Q \leq 100$ the results were fitted to a power law $S(Q) \propto Q^{-\gamma}$. The numerical work was carried out on a HP Apollo 715/33 workstation with typical CPU times of about $20000 \mathrm{~s}$ per run. For $H=0.3$ values of $\gamma=2.591,2.568,2.549 \pm 0.001$ were obtained for the different parameters of $\left(\left|q_{z}^{\prime}\right| \sigma\right)^{2}$, respectively, see Fig. 6 . In the case of $H=0.5$ the result was $\gamma=2.994,2.990$, $2.988 \pm 0.001$, respectively, and for $H=0.8, \gamma=2.602$, $3.6128,3.623 \pm 0.001$. Thus, the Fourier transform is a good approximation (i.e., the roughness is called weak) for $\left(\left|q_{z}^{\prime}\right| \sigma\right)^{2} \leq 0.1$, but can also be used for higher values if $H \geq 0.5$, depending on the required accuracy in the determination of $H$. As an example, at $\sigma=5 \AA, \alpha_{i, f} \leq 0.5^{\circ}$ and $\lambda=1.5 \AA$ the condition of weak roughness is clearly fulfilled. Moreover, even at much higher values of $\sigma$ this 
approximation is correct, if $\alpha_{i, f} \simeq \alpha_{c}$, as the normal momentum transfer in the medium is minimized in this case, see Fig. 4. A scattering geometry with $\alpha_{i, f} \simeq \alpha_{c}$ will be discussed in the next section.

Therefore it is of practical interest to make use of the free choice in the form of $c(r)$ and select the $K$ correlation function with its analytical Fourier transform, Eq. (2.18). Thus, in the "Fourier transform approximation," the exponent $\gamma$ governing the intensity decay and the roughness exponent $H$ is given by the following analytical expression:

$$
\gamma=2+2 H
$$
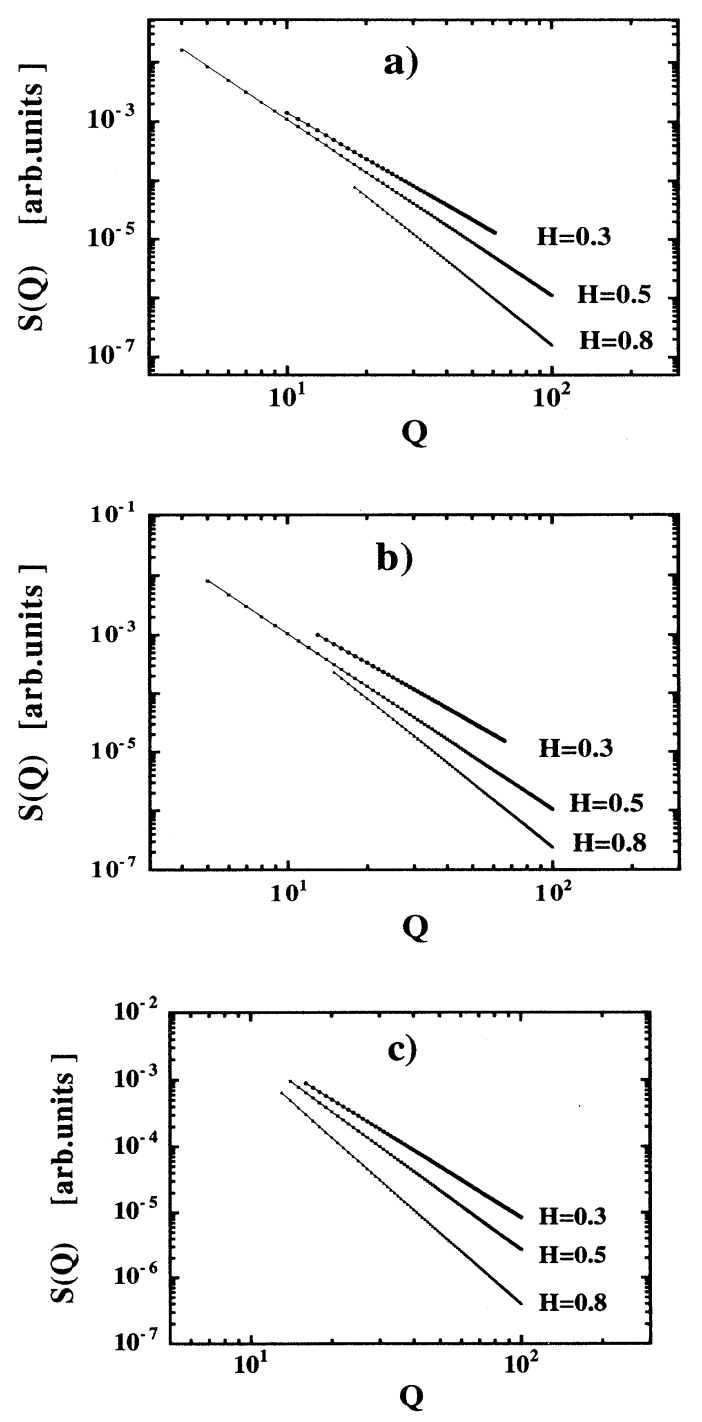

FIG. 6. Numerical calculation of the structure factor using the correlation function according to $\mathrm{Eq}$. (2.16) for different values of $\left(\left|q_{z}^{\prime}\right| \sigma\right)^{2}$. The solid lines correspond to a least-squares fit to the power $\gamma$. (a) $\left(\left|q_{z}^{\prime}\right| \sigma\right)^{2}=0.1$, (b) $\left(\left|q_{z}^{\prime}\right| \sigma\right)^{2}=0.5$, (c) $\left(\left|q_{z}^{\prime}\right| \sigma\right)^{2}=1$.
Apart from the intensity decay with the parallel momentum transfer $Q$, the static roughness exponent $H$ can also be determined from the diffuse intensity decay along $q_{z}$. At first sight, it may seem surprising that a quantity describing lateral correlations enters in $S\left(Q=0, q_{z}^{\prime}\right)$. However, this is a manifestation of the fact that $S(q)$ in Eq. (3.3) is not exactly the Fourier transform of $c(r)$. Accordingly, at $\left(\left|q_{z}^{\prime}\right| \sigma\right)^{2} \leq 1$, where the approximation holds, $S\left(q_{z}^{\prime}, Q=0\right)$ is roughly constant, but falls off with a power of $2+2 / H(2+1 / H$ in the case of wide open slits) for $\left(\left|q_{z}^{\prime}\right| \sigma\right)^{2} \geq 1$. This can be checked easily, e.g., for the case of wide open slits, as the integrand in Eq. (3.4) with $c(r)$ given by Eq. (2.11) can be expanded in a Taylor series for $Q=0$, every term of which can be integrated to give

$$
\begin{aligned}
S\left(Q=0, q_{z}^{\prime}\right)= & \frac{\exp \left[-\operatorname{Re}\left(q_{z}^{\prime}\right)^{2} \sigma^{2}\right]}{\left|q_{z}^{\prime}\right|^{2}} 2 \xi \Gamma(1+1 / 2 H) \\
& \times \sum_{n=1}^{\infty} \frac{\left(\left|q_{z}^{\prime}\right| \sigma\right)^{2 n}}{n ! n^{1 / 2 H}}
\end{aligned}
$$

which can be computed easily. The result is shown in Fig. 7 for $H=0.3,0.5,0.8$. However, it is not clear whether the power-law decay of $S\left(Q=0, q_{z}^{\prime}\right)$ is independent of the function $c(r)$. Unlike for the exponent $\gamma$ in Eq. (3.6) we do not have a fundamental theorem on the asymptotic behavior of Fourier transformations at hand to answer this question.

At the end of this section a few remarks should be made concerning further contributions to nonspecular scattering, that stem from lateral disorder other than interfacial roughness. As known, amorphous structures lead to isotropical scattering, that can be described by a structure factor $S_{\text {bulk }}(|q|)$, reflecting the atomic correlation of the amorphous material. $S_{\text {bulk }}(|\mathbf{q}|)$ rests finite over the whole $q$ range, converging to a constant value for low $|\mathbf{q}|$ :

$$
S_{\text {bulk }}(0)=k_{B} T \rho \chi_{T}
$$

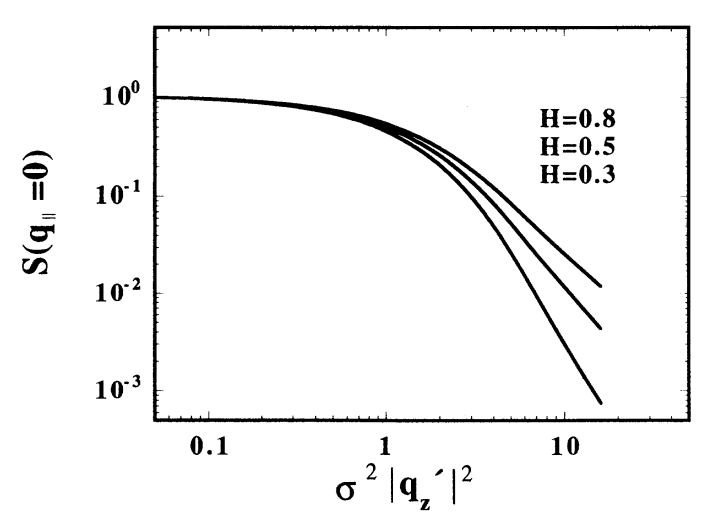

FIG. 7. Structure factor $S\left(\left|q_{z}^{\prime}\right|, Q=0\right)$ at constant parallel momentum transfer calculated from Eq. (2.12) as a function o $\left(\left|q_{z}^{\prime}\right| \sigma\right)^{2}$ for different values of $H$. 
where $\rho$ denotes the density of scatterers and $\chi_{T}$ the isothermal compressibility. ${ }^{13}$ It is an experimentally established fact, that $S(|\mathbf{q}|)$ stays practically constant (within a few percent) in the range $0 \leq|\mathbf{q}| \leq q_{\max } / 4$, if $q_{\max }$ is the position of the first amorphous maximum. ${ }^{13}$ In contrast to this smooth behavior of $S_{\text {bulk }}(|q|)$, the structure factor of a rough interface $S(q)$ increases rapidly at low $q_{\|}$, so that the two different contributions can be distinguished by their drastically different $q$ dependence. ${ }^{14}$ At small angles the scattering intensity due to the amorphous state is a negligible background to the nonspecular intensity resulting from interfacial roughness. However, this is only true as long as there are no density fluctuations on long length scales $r \gg a$ in the bulklike in porous media for example. In this case, the two sources of nonspecular scattering can be distinguished only by the intensity distribution as a function of $q_{z}{ }^{15}$

\section{SCATTERING GEOMETRY}

As discussed in Sec. I, self-affine roughness is defined by the scaling behavior of the height difference function $g(r)$ [or the height-height correlation function $c(r)$ ] for spatial distances smaller than the cutoff length, $r \leq \xi$. Consequently, if the spatial roughness exponent $H$ is to be determined from an x-ray scattering experiment, a region in reciprocal space sensitive to these correlation length scales should be sampled. Otherwise the data fitting will give a meaningless value that depends strongly on the special functional form $c(r)$ chosen to model the data but does not characterize the scaling behavior of the interface. As we have shown in the previous section, a reliable determination of $H$ requires measuring the diffuse intensity at $q_{z} \geq 2 \pi / \sigma$ or $q_{\|} \leq 2 \pi / \xi$.

The standard geometries to record nonspecular data are the so-called rocking scan, the detector scan, and the offset scan. ${ }^{2-4}$ In the rocking scan, the detector is held fixed, while the sample is rocked around an axis normal to the plane of reflection, so that the sum of the incidence and exit angle is constant. In this case, a small change, in $\alpha_{i}=\alpha \pm \Delta \alpha$ leads to the following change in momentum transfer:

$$
\begin{aligned}
\Delta q_{x} & = \pm \frac{4 \pi}{\lambda} \sin \alpha \sin (\Delta \alpha) \simeq \pm \frac{4 \pi}{\lambda} \alpha \Delta \alpha \\
\Delta q_{z} & =-\frac{4 \pi}{\lambda}[\sin \alpha \cos (\Delta \alpha)-\sin \alpha] \\
& \simeq-\frac{2 \pi}{\lambda} \alpha(\Delta \alpha)^{2}
\end{aligned}
$$

In the detector scan the sample is held fixed, and the detector position is varied around the specular. Changing the exit angle will mainly result in a change of $q_{z}$ and only a very small change of $q_{z}$, according to

$$
\begin{aligned}
& \Delta q_{x}=-\frac{2 \pi}{\lambda} \sin \alpha \sin (\Delta \alpha) \simeq-\frac{2 \pi}{\lambda} \alpha \Delta \alpha, \\
& \Delta q_{z}=-\frac{2 \pi}{\lambda} \cos \alpha \sin (\Delta \alpha) \simeq-\frac{2 \pi}{\lambda} \Delta \alpha .
\end{aligned}
$$

The offset scan is essentially the same scan as that of specular reflectivity $\alpha_{i}=\alpha_{f}$, except that the $\alpha_{i}$ calibra- tion is intentionally offset by an fixed angle $\Delta \alpha_{i}$.

Recently, we have applied the geometry of grazing incidence diffraction to the field of nonspecular scattering from rough interfaces. ${ }^{16}$ However, in order not to confuse this method with an actual diffraction experiment where scattering is studied around a Bragg peak, ${ }^{17}$ we refer to this geometry as the out-of-plane scan, because the detector is moved out of the plane of reflection by an angle $2 \theta$, while the incidence and exit angles $\alpha_{i}$ and $\alpha_{f}$, respectively, are kept constant, as shown in Fig. 8. The q vector is then given by

$$
\begin{aligned}
& q_{x}=\frac{2 \pi}{\lambda}\left(\cos \alpha_{f} \cos 2 \theta-\cos \alpha_{i}\right), \\
& q_{y}=\frac{2 \pi}{\lambda} \sin 2 \theta \cos \alpha_{f}, \\
& q_{z}=\frac{2 \pi}{\lambda}\left(\sin \alpha_{f}+\sin \alpha_{i}\right),
\end{aligned}
$$

and a variation of $2 \theta$ around zero gives

$$
\Delta q_{y}= \pm \frac{2 \pi}{\lambda} \sin (\Delta 2 \theta) \simeq \pm \frac{2 \pi}{\lambda} \Delta 2 \theta \text {. }
$$

At large angles $2 \theta$, i.e., around a Bragg peak or a maximum of the amorphous structure factor, atomic correlations can be probed, whereas in the range $q_{\|} \ll 1 \AA^{-1}$ the scattering intensity is expected to be dominated by the structure factor of a rough surface, Eq. (3.3).

At small $q_{\|}$, the nonspecular intensity close to the specular position is difficult to separate from the specularly reflected beam. The incident beam of any real x-ray source has a finite divergence that is reflected from the rough surface to lowest order like from a planar mirror, thereby contaminating the pure nonspecular scattering due to the roughness. These intensity tails of the primary beam have to be deconvoluted like it is usually done in small angle scattering experiments to obtain correct results. For momentum transfer smaller than $0.006 \AA^{-1}$ corresponding to correlation lengths larger than $\xi \simeq 1000 \AA$ special conditions have to be met to suppress

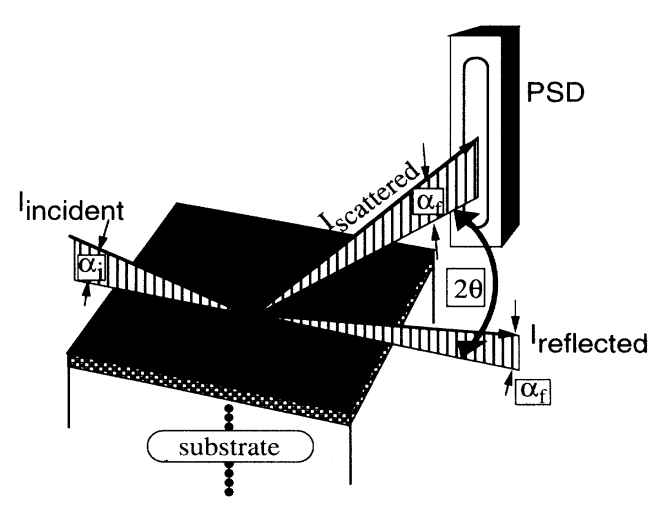

FIG. 8. Scattering geometry of grazing incidence diffraction, with a one-dimensional position-sensitive detector (PSD) oriented parallel to the surface normal. 
scattering from air and imperfect slits to become necessary, if the nonspecular intensity is not particularly high. The primary beam should be tuned to be especially narrow by setting small perfect slits parallel to the scattering plane and residual scattering by air should be suppressed by evacuation of the beam path.

However, the scattering geometry can by itself have a drastic influence on how well defined the primary beam has to be. For scans in the plane of reflection like the rocking or detector scan at grazing incidence a certain increase in the angle of incidence $\Delta \alpha$ corresponds only to a small increase in $\Delta q_{x}$, see Eqs. (4.1)-(4.3), so that the reciprocal space is "affected" by the tails of the specular beam only in a very small range of $q_{x}$ around the origin. Thus, in contrast to the transmission geometry that is used in most small angle scattering experiments, much larger real-space structures $d \simeq 10 \mu \mathrm{m}$ can be probed in reflection geometry at small angles of incidence and exit. That is the reason why most nonspecular experiments can be performed without the usual small-angle requirements. This argument, however, does not apply to the out-of-plane scan, where $\Delta q_{y}$ changes rapidly with the scattering angle, see Eq. (4.8). The out-of-plane geometry is therefore best suited for the spatial length scales $10 \AA \leq 1000 \AA$, where the conventional geometries are less appropriate, because they are strongly limited in the accessible range of $q_{\|}$. In a rocking scan, the incidence and exit angles $\alpha_{i}$ and $\alpha_{f}$ can only be varied within the limits given by the sample surface. In a detector scan, $\alpha_{f}$ can be increased without any constraint of the sample surface, but a change in $\alpha_{f}$ gives only a slight increase in $q_{\|}$, while at the same time a strong intensity decay is observed due to the quickly increasing $q_{z}$, see Eq. (4.8).

The restrictions due to the sample surface in the conventional in-plane scattering geometries are sketched in Fig. 9. The semicircles indicate the limits due to the sample surface, that are typical for scans in the plane of reflection. Experimentally, the high- $q_{z}$ region is difficult to measure due to the strong decay in intensity, see Eq. (3.9). It is therefore of advantage to measure at low $q_{z}$. The $q_{\|}$range accessible in the out-of-plane scattering

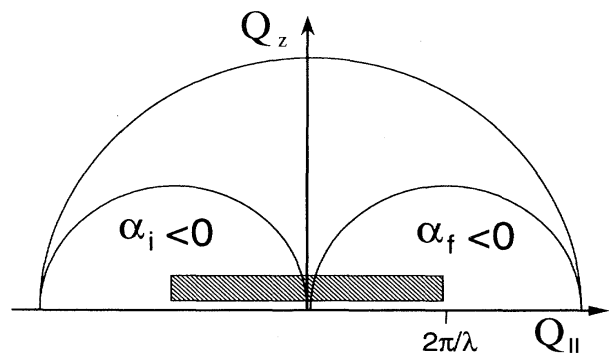

FIG. 9. The Ewald semicircle: Only the lightly hatched area is accessible if the detector is kept in the plane of reflection, i.e., at small angles of incidence and exit angles the parallel momentum transfer $q_{\|}$is significantly limited by the sample surface. The darkly hatched area shows the area probed in reciprocal space by a single out-of-plane scan with a PSD. geometry is indicated as the darkly hatched area.

It is of further disadvantage in the conventional geometries that the Fresnel transmission functions, Eq. (3.2), strongly influence the measured intensity (e.g., Yoneda peak), whereas they enter the out-of-plane geometry solely in the form of an overall scaling factor. Thus, data evaluation is more straightforward in this case and experimental errors can be kept smaller.

For comparison, the nonspecular intensity as measured in a rocking, detector, and out-of-plane scan are shown for the same set of parameters $\lambda=1.55 \AA, \sigma=5 \AA$, $\xi=5000,500 \AA$, and $H=0.3,0.5,0.8$, in Figs. 10-12, respectively. The refractive index in the medium is given by $n=1-1.5 \times 10^{-5}-i 7.5 \times 10^{-7}$. In all cases the specular condition is given by $\alpha_{i}=\alpha_{f}=0.5^{\circ}$, but the specular peak has been omitted as to discuss solely the nonspecular intensity. The curves have been calculated using $c(r)$ as given by Eq. (2.11) and have been normalized to their maximum values, respectively. For the in-plane scans the intensity has been integrated over the out-of-plane direction, which corresponds to the so-called open slit measurement. As can be seen, the rocking curves for $\xi=500 \AA$ are not at all sensitive to the parameter $H$. The corresponding curves of the detector scan start to split up
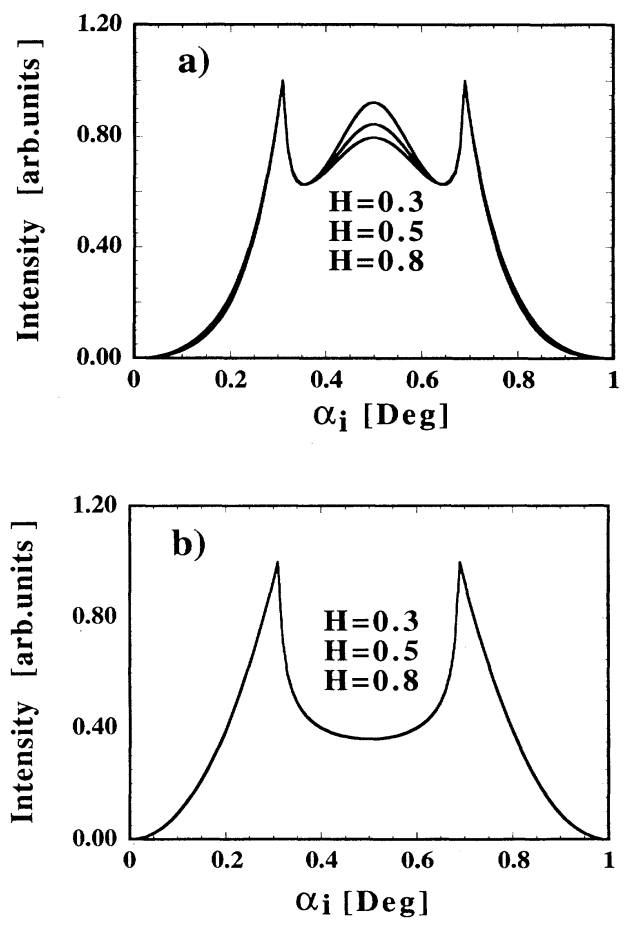

FIG. 10. Simulation of the diffuse intensity (normalized to its peak value) of a rough surface with $\sigma=5 \AA$, and (a) $\xi=5000 \AA$, (b) $\xi=500 \AA$ for $H=0.3,0.5,0.8$ (from top to bottom), respectively, as measured in a rocking scan at $\alpha_{f}=0.5^{\circ}$ on a linear scale. Two Yoneda peaks at $\alpha_{i, f}=\alpha_{c}$, respectively, are observed. In (a) the curves corresponding to different $H$ can be distinguished, while in (b) all three curves lie on top of each other. 

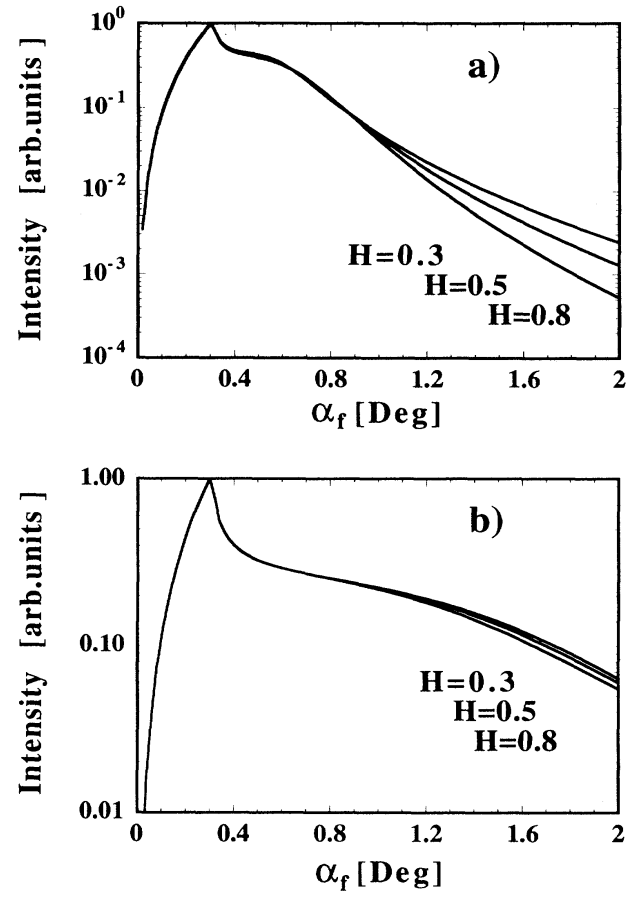

FIG. 11. Same as Fig. 10 for a detector scan on a semilog scale, at $\alpha_{i}=0.5^{\circ}$. (a) At high values of $\alpha_{f}$ a good sensitivity on $H$ is observed. Note the order of magnitude of the intensity decay before the curves split. (b) For $\xi=500 \AA$ only a slight sensitivity on $H$ is observed.
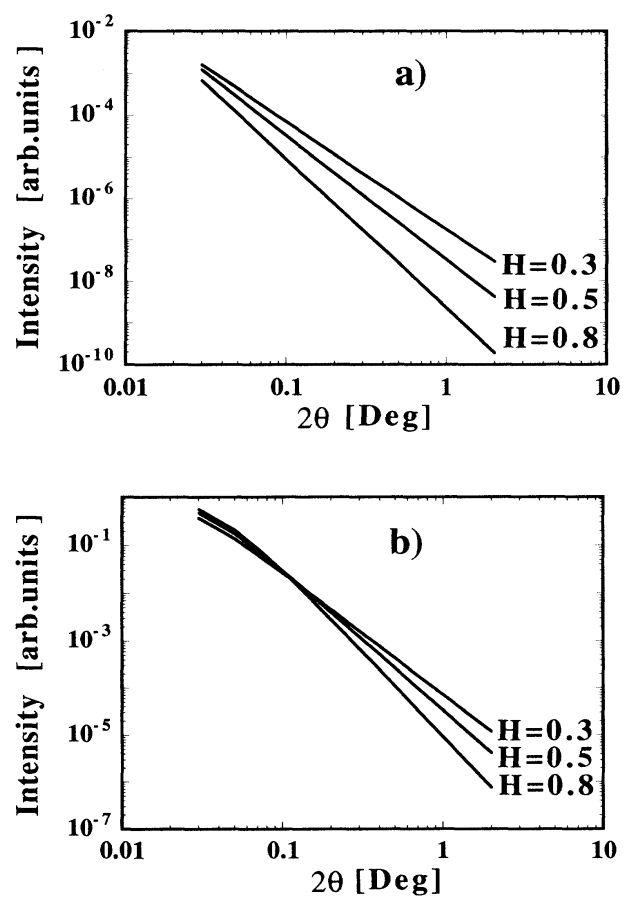

FIG. 12. Same as Fig. 10 for an out-of-plane scan on a loglog scale. Here, in both (a) and (b) the curves have an obviously different slope. Note the lack of any Yoneda peak due to the constant incidence and exit angles. slightly at large $\alpha_{f}$. This is due to the limited range in $q_{\|}$ in these scans. In fact, in the detector scan the parallel momentum transfer is limited to $0.0023 \AA^{-1}$ at $\alpha_{f}=2^{\circ}$, i.e., $Q:=q_{\|} \xi=1.16$ and $Q=11.58$, for $\xi=500 \AA$ and $\xi=5000 \AA$, respectively, whereas the out-of-plane scan reaches a value of $q_{\|}=0.14 \AA^{-1}$ at $2 \theta=2^{\circ}$. In a rocking or detector scan one will therefore probe the plateau of the structure factor, but not the part of the power-law decay which is significant for the determination of $H$, unless $\xi$ is sufficiently high. In the case of $\xi=5000 \AA$ the rocking curves can be distinguished only in the central part, where the nonspecular intensity might be hidden under the slopes of the specular beam, if the divergence (as measured on a logarithmic scale) is not particularly small.

In summary, the out-of-plane scan is capable of probing real-space correlations in the whole range down to atomic distances, whereas the maximum value reported in an in-plane geometry was $0.06 \AA^{-1}$ for a multilayer sample measured in a rocking scan with the specular position at the fifth Bragg peak. ${ }^{4}$ On the other hand, in the plane of reflection the resolution in $q_{\|}$is much higher and large correlation lengths $\xi \simeq 10000 \AA$ can be determined easily. As we have discussed in the previous sections, the determination of $H$ requires measuring $c(r)$ at $r \leq \xi$, i.e., measuring at $q_{\|} \geq 2 \pi / \xi$, so that a suitable scattering geometry must be chosen according to the value of $\xi$.

\section{MULTILAYERS}

Synthetic multilayers are made of alternating layers of chemically different materials that are usually grown from the vapor phase by elaborated techniques like molecular beam epitaxy (MBE), electron beam evaporation, magnetron sputtering, or laser ablation. Such systems with a nanometer periodicity $d$ in the growth direction are of high interest for various applications in optoelectronic devices, in supermirrors for neutrons or Bragg mirrors in soft $x$-ray optics. ${ }^{18}$ The performance depends crucially on the roughness of the interfaces and special efforts are made to control the relevant parameters, e.g., substrate temperature, flux, energy of sputtering ions, etc., to suppress the kinetic roughening considerably during the growth process.

In contrast to a single rough layer, additional correlations across different interfaces must be expected, the socalled height-height cross correlations $c_{i j}(r):=\left\langle h_{i}(\mathbf{r}) h_{j}\left(\mathbf{r}^{\prime}\right)\right\rangle$, where $i, j$ denote two different interfaces. A multilayer with perfect cross correlations, i.e., where the fluctuations of one layer are copied throughout the stack, is called conformal. If one succeeds to determine these cross correlations experimentally, access not only to the spatial, but also to the temporal aspects of the growth process is obtained, as different interfaces can be attributed to different times $t$ during growth. ${ }^{19}$ Recently, both the height-height selfand cross-correlation functions determined by nonspecular $\mathrm{x}$-ray scattering from a W/Si magnetron sputtered multilayer have been shown to agree with those predicted by the Edwards-Wilkinson growth equation. ${ }^{16}$

In this section, we want to focus on how the height- 
height self-correlation functions averaged over the top $N$ interfaces can be measured. $N$ is limited by the usual scattering depth of grazing incidence diffraction. ${ }^{17}$ For a periodic multilayer made up of two materials $A$ and $B$ with absorption coefficients and layer thicknesses, $\mu_{A}$, $\mu_{B}$, and $d_{A}, d_{B}$ respectively, with both the incidence and exit angles greater than the critical angle $N$ will be given by

$$
N=\frac{2 \ln 0.5}{\left(\mu_{A} d_{A}+\mu_{B} d_{B}\right)\left(\sin \alpha_{i}+\sin \alpha_{f}\right)}
$$

as long as the Bragg condition of the multilayer $\alpha_{i, f}=\alpha_{n}=\arcsin (n \lambda / 2 d)$ is not fulfilled. In the latter case the reflected amplitudes at each interface interfere constructively and extinction can thus limit the penetration depth independently of absorption.

According to kinetic roughening theory, all $N$ interfaces are assumed to have the same static roughening exponent. However, for the cutoff length of the $i$ th interface, $\xi_{i}$, two different scenarios are possible: (a) The growth has become stationary for all interfaces probed, i.e., the $\xi_{i}$ 's are all the same. (b) $\xi_{i}$ is a growing function of $i$, and by taking advantage of the proportionality between thickness and time, $\xi_{i} \propto(i d)^{1 / z}$. However, also in the latter case we can consider fluctuations of small length scales that have already become stationary. Thus, if we measure at lateral momentum transfer $q_{\|} \ll 1 / \xi_{i}$, we see $N$ statistically equivalent interfaces. If the roughness of the different interfaces was uncorrelated, i.e., the cross-correlation functions were zero, the scattered intensity would add up incoherently, and in any scattering geometry we would measure just $N$ times the scattering cross section of one such interface. The more general case of partially correlated interfaces leads to a modulation of the intensity along $q_{z}$ with the positions of the maxima given by $q_{z}=2 \pi / d$, the so-called Bragg sheets. The intensity scattered in these Bragg sheets stems from correlated roughness components, whereas the intensity in between two sheets results from uncorrelated fluctuations.

The calculation of the multilayer diffuse scattering has first been solved for the limiting case of weak roughness, ${ }^{20}$ and later for the more general case of Eq. (3.1) in the framework of the DWBA for the case of uncorrelated $^{21}$ and correlated interfaces, ${ }^{22}$ respectively. In all cases a semidynamical approach was chosen: The reflected and transmitted waves in the multilayer stack were treated dynamically by the application of a matrix method or Parratt algorithm, ${ }^{23}$ whereas the diffuse scattering was treated kinematically, i.e., multiple scattering was neglected. This approximation is believed to hold as long as the total amount of nonspecular intensity is small compared to the specularly reflected intensity, ${ }^{20}$ which always holds for small angles of incidence and exit. The implementation of the calculation schemes cited above in a computer simulation program allows one, in principle, to analyze experimental data and to determine the roughness exponent. However this requires the assumption of a specific model for the cross correlations.

An alternative method that has been applied in Ref. 16 is the integration of the scattered intensity over one Brillouin zone of the multilayer $\Delta q_{z}=2 \pi / d$, in a way that coherent and uncoherent contributions to the intensity are equally probed, and the result is just $N$ times the intensity of one interface. In this case no model of specific cross-correlation functions has to be assumed. Instead, one can use the simple Eq. (3.1) derived for a single interface to analyze the multilayer sample. Experimentally, the integration along $q_{z}$ can be most easily implemented by the use of a position-sensitive detector (PSD) directed parallel to the surface normal in an out-of-plane scan. At small exit angles, the variation of $\mathbf{q}$ with the detector channels is then mainly one in $q_{z}$ and only to a negligible part in $q_{x}$, see Eqs. (4.3) and (4.4). Let the PSD be centered on a Bragg sheet $q_{z}^{B}=n 2 \pi / d$ with the upper and lower end of the PSD extending to the upper and lower zone boundary $n 2 \pi / d \pm \pi / d$, respectively. The integrated intensity can then be plotted on a log-log scale as a function of $q_{\|}(2 \theta)$ to determine the exponent $\gamma$, just like in the case of a single surface.

As was discussed in Sec. II, in the limit of weak roughness $\left(\left|q_{z}^{\prime}\right| \sigma\right)^{2} \ll<1$ the exponent $\gamma$ to be determined is independent of $\left(\left|q_{z}^{\prime}\right| \sigma\right)^{2}$. Generally this is not the case and the decay of the intensity with $q_{\|}$depends on the particular $q_{z}$ position in the Brillouin zone. The integration will hence introduce an error if the width of the Bragg sheet is not small compared to the width of the Brillouin zone. Let $I\left(q_{z}-q_{z}^{B}\right)$ be the normalized intensity distribution along $q_{z}$ at a particular $q_{\|}$. If the interface fluctuations of the corresponding wavelength are correlated, this function will have a sharp peak at the position of the Bragg sheet. If the fluctuations are uncorrelated, it will be flat. In the asymptotic regime $Q_{\|} \geq 1$, where $\gamma$ is to be determined, the intensity decay with $Q_{\|}$is then proportional to

$$
S\left(Q_{\|}\right) \propto \int_{-\pi / d}^{\pi / d} d q_{z} \frac{I\left(q_{z}-q_{z}^{B}\right)}{Q_{\|}^{\gamma}},
$$

where $\gamma$ is a function of both $H$ and $q_{z}$, e.g., in the case of a logarithmic correlation function $\gamma=2+\left(\sigma q_{z}\right)^{2}$, see Eq. (3.7).

\section{SUMMARY}

We have discussed the method of diffuse x-ray scattering as applied to self-affine interfaces. The basic assumptions leading to the prediction of such interface structures have been summarized in order to elucidate the fact that only the asymptotical behavior of the height-height correlation function is predicted, rather than the entire function. The properties of the correlation function for distances much smaller than the upper cutoff can be measured at high parallel momentum transfer. If the correlation function decays by a power of $2 H$ in this range, the scattered intensity will also fall off by a power law with parallel momentum transfer. From the corresponding exponent $\gamma$ the static scaling exponent can be deduced. We have presented an analytical approximation for $\gamma$ valid for the case of "weak roughness," where the structure factor can be approximated by the Fourier transform of 
the correlation function. This approximation was checked by numerical calculations and was found to hold in a wider range than commonly believed. Therefore, frequently experimental data need not be evaluated by tedious numerical fitting. This should be especially interesting for multilayer samples where the necessary calculation time can easily exceed available CPU time. Different nonspecular scan types were discussed with respect to their sensitivity to $H$. It was found that for upper cutoff lengths smaller than $\simeq 1000 \AA, H$ can only be determined from the diffuse scattering as a function of $q_{z}^{\prime}$ at $q_{\|} \simeq 0$ (offset scan) or from the exponent $\gamma$ by means of an out- of-plane scan. Finally, it was shown how the static scaling exponent in an multilayer system can be determined without any knowledge or model of the cross-correlation functions between different interfaces.

\section{ACKNOWLEDGMENTS}

This work was supported by the Bundesministerium für Forschung und Technologie under Contract No. 055WMAXI. We thank H. Spohn for fruitful discussions, and S. Vitta for a careful reading of the manuscript.
${ }^{1}$ S. K. Sinha et al., Phys. Rev. B 38, 2297 (1988).

${ }^{2}$ W. Weber and B. Lengeler, Phys. Rev. B 46, 7953 (1992).

${ }^{3}$ D. E. Savage et al., J. Appl. Phys. 69, 1411 (1991).

${ }^{4}$ Y. H. Phang et al., J. Appl. Phys. 74, 3181 (1993), and references therein.

${ }^{5}$ For an overview see J. Krug and H. Spohn, in Solids far from Equilibrium, edited by C. Godrèche (Cambridge University Press, Cambridge, 1992).

${ }^{6}$ G. Palasantzas and J. Krim, Phys. Rev. B 48, 2873 (1993).

${ }^{7}$ For an overview see P. Meakin, Phys. Rep. 235, 191 (1993).

${ }^{8}$ M. Kardar, G. Parisi, and Y. C. Zhang, Phys. Rev. Lett. 56, 889 (1986).

${ }^{9}$ B. M. Forrest and L. Tang, J. Stat. Phys. 60, 181 (1990).

${ }^{10}$ G. Palasantzas, Phys. Rev. B 48, 14472 (1993).

${ }^{11}$ Y. Yoneda, Phys. Rev. 131, 2010 (1963).

${ }^{12}$ W. Weber, Ph.D. thesis RWTH Aachen, 1992.

${ }^{13} \mathrm{Y}$. Waseda, The Structure of Non-Crystalline Materials
(McGraw-Hill, New York, 1980), p. 234.

${ }^{14}$ T. Salditt et al., J. Phys. III (France) 4, 1573 (1994).

${ }^{15} \mathrm{M}$. Rauscher, T. Salditt, and H. Spohn (unpublished).

${ }^{16}$ T. Salditt, T. H. Metzger, and J. Peisl, Phys. Rev. Lett. 73, 2228 (1994).

${ }^{17}$ H. Dosch, Critical Phenomena at Surfaces and Interfaces, Springer Tracts in Modern Physics Vol. 126B (Springer, Berlin, 1992).

${ }^{18}$ P. Dhez and C. Weisbuch, Physics, Fabrication, and Applications of Multilayered Structures, Vol. 182 of NATO Advanced Study Institute, Series B: Physics (Plenum, New York, 1988).

${ }^{19}$ D. G. Stearns, Appl. Phys. Lett. 62, 1745 (1993).

${ }^{20}$ D. G. Stearns, J. Appl. Phys. 71, 4286 (1992).

${ }^{21}$ H. Holý et al., Phys. Rev. B 47, 15896 (1993).

${ }^{22}$ V. Holý and T. Baumbach, Phys. Rev. B 49, 10668 (1994).

${ }^{23}$ L. G. Parratt, Phys. Rev. 95, 359 (1954). 